\title{
СХОДИМОСТЬ АЛГОРИТМА АДДИТИВНОЙ РЕГУЛЯРИЗАЦИИ ТЕМАТИЧЕСКИХ МОДЕЛЕЙ ${ }^{1}$
}

\author{
И. А. Ирхин, К. В. Воронцов
}

\begin{abstract}
Задача вероятностного тематического моделирования заключается в следующем. По заданной коллекции текстовых документов требуется найти условное распределение каждого документа по темам и условное распределение каждой темы по словам (или термам). Для решения данной задачи используется принцип максимума правдоподобия. Задача имеет в общем случае бесконечное множество решений, т. е. является некорректно поставленной по Адамару. В рамках подхода ARTM - аддитивной регуляризации тематических моделей к основному критерию добавляется взвешенная сумма нескольких дополнительных критериев регуляризации. Численный метод для решения данной задачи - разновидность итерационного ЕM-алгоритма, который выписывается в общем виде для произвольного гладкого регуляризатора, в том числе и для линейной комбинации гладких регуляризаторов. В работе исследуется вопрос о сходимости данного итерационного процесса. Получены достаточные условия сходимости, при которых процесс сходится к стационарной точке регуляризованного логарифма правдоподобия. Полученные ограничения на регуляризатор оказались не слишком обременительными. В работе даны их интерпретации с точки зрения практической реализации алгоритма. Предложена модификация алгоритма, которая улучшает его сходимость без дополнительных затрат времени и памяти. В экспериментах на коллекции новостных текстов показано, что данная модификации позволяет не только ускорить сходимость, но и улучшить значение оптимизируемого критерия.
\end{abstract}

Ключевые слова: обработка текстов естественного языка, вероятностное тематическое моделирование, вероятностный латентный семантический анализ (PLSA), латентное размещение Дирихле (LDA), аддитивная регуляризация тематических моделей (ARTM), EM-алгоритм, достаточные условия сходимости.

I. A. Irkhin, K. V. Vorontsov. Convergence of the algorithm of additive regularization of topic models.

The problem of probabilistic topic modeling is as follows. Given a collection of text documents, find the conditional distribution over topics for each document and the conditional distribution over words or terms for each topic. Log-likelihood maximization is used to solve this problem. The problem has generally an infinite set of solutions, being ill-posed according to Hadamard. In the framework of Additive Regularization of Topic Models (ARTM), a weighted sum of regularization criteria is added to the main log-likelihood criterion. The numerical method for solving this optimization problem is a kind of iterative EM-algorithm. In ARTM it is inferred in a quite general form for an arbitrary smooth regularizer, as well as for a linear combination of smooth regularizers. This paper studies the problem of convergence of the EM iterative process. Sufficient conditions are obtained for the convergence to a stationary point of the regularized log-likelihood. The constraints imposed on the regularizer are not too restrictive. We give their interpretations from the point of view of the practical implementation of the algorithm. A modification of the algorithm is proposed that improves the convergence without additional time and memory costs. Experiments on the news text collection have shown that our modification both accelerates the convergence and improves the value of the criterion to which it converges.

Keywords: natural language processing, probabilistic topic modeling, probabilistic latent semantic analysis (PLSA), latent Dirichlet allocation (LDA), additive regularization of topic models (ARTM), EM-algorithm, sufficient conditions for convergence.

MSC: 90C30, 68T50

DOI: $10.21538 / 0134-4889-2020-26-3-56-68$

\footnotetext{
${ }^{1}$ Работа выполнена в рамках проекта "Средства интеллектуального анализа больших массивов текстов" по Программе ЦК НТИ “Центр хранения и анализа больших данных", поддерживаемого Министерством науки и высшего образования Российской Федерации по договору МГУ им. М. В. Ломоносова с Фондом поддержки проектов НТИ от 15.08.2019 № 7/1251/2019. Работа также частично поддержана РФФИ, проект 20-07-00936.
} 


\section{Введение}

Тематическое моделирование - одно из современных направлений обработки естественного языка (natural language processing, NLP). Тематическая модель коллекции текстовых документов определяет, к каким темам относится каждый документ и какие термы образуют каждую тему. Термами могут быть слова, нормальные формы слов, словосочетания или термины - в зависимости от того, какие виды предварительной обработки текста были применены к данной коллекции. Тематическое моделирование не претендует на полноценное понимание естественного языка (natural language understanding, NLU), однако выявление тематики текстов можно считать определенным шагом в этом направлении.

Вероятностная тематическая модель (probabilistic topic model, PTM) описывает каждый документ дискретным распределением вероятностей на множестве тем, каждую тему - дискретным распределением вероятностей на множестве термов. Построенная модель позволяет преобразовать любой текст в вектор вероятностей тем. Важным преимуществом тематического векторного представления текста является его интерпретируемость. Каждая координата вектора показывает долю соответствующей темы в тексте, при этом семантика темы описывается частотным словарем термов, т. е. фактически словами естественного языка.

Тематическое моделирование, как и кластеризация документов, относится к методам обучения без учителя и не требует какой-либо разметки текстов или экспертных оценок. Отличие в том, что при кластеризации документ целиком относится к одному кластеру, тогда как тематическая модель осуществляет мягкую кластеризацию (soft clustering), разделяя документ между несколькими кластерами-темами. Тематические модели называют еще моделями мягкой би-кластеризации, поскольку термы также кластеризуются по темам. Это позволяет обходить проблемы синонимии и полисемии слов. Синонимы, употребляемые в схожих контекстах, группируются в одних и тех же темах. Многозначные слова и омонимы, наоборот, распределяют свои вероятности по нескольким семантически не связанным темам.

Перечисленные особенности вероятностного тематического моделирования делают его важным инструментом семантического анализа больших текстовых коллекций.

Построение тематической модели по коллекции документов является некорректно поставленной оптимизационной задачей приближенного стохастического матричного разложения, которая в общем случае имеет бесконечное множество решений. Согласно теории регуляризации А. Н. Тихонова (см. [9]) решение такой задачи возможно доопределить и сделать устойчивым. Для этого к оптимизационному критерию добавляется регуляризатор - дополнительный критерий, учитывающий специфические особенности прикладной задачи или знания предметной области. В сложных приложениях дополнительных критериев может быть несколько.

Аддитивная регуляризаиия тематических моделей (additive regularization of topic models, ARTM) - это многокритериальный подход, в котором для оптимизации параметров модели используется взвешенная сумма критериев (см. $[11 ; 14 ; 15])$. ARTM позволяет строить модели с требуемыми свойствами, суммируя регуляризаторы, исходно предлагавшиеся в различных моделях, главным образом в рамках байесовского обучения (см. [6]). Однако в байесовском обучении не существует общего подхода к комбинированию регуляризаторов от разных моделей. В ARTM для обучения модели с произвольной линейной комбинацией регуляризаторов используется один и тот же EM-подобный алгоритм (EM-like algorithm), при этом для добавления нового регуляризатора достаточно знать его частные производные по параметрам модели. Это приводит к модульной технологии тематического моделирования, которая реализована в библиотеке с открытым кодом BigARTM, http://bigartm.org (cм. [4;12]).

Подчеркнем, что ARTM не является еще одной частной тематической моделью или методом - это общий подход к построению и комбинированию тематических моделей.

До сих пор в теории ARTM оставались открытыми вопросы о сходимости EM-алгоритма и о влиянии регуляризаторов на сходимость. В данной статье показано, что в ARTM итерации ЕМ-алгоритма возможно интерпретировать как итерации обобщенного ЕМ-алгоритма 
(Generalized EM, GEM; см. [3]), для которого условия сходимости хорошо изучены (см. [17]). В работе получены ограничения на регуляризаторы, обеспечивающие сходимость, и предложена модификация ЕМ-алгоритма, улучшающая его сходимость.

\section{1. Задача тематического моделирования с аддитивной регуляризацией}

Пусть $D$ - конечное множество (коллекция) текстовых документов, $W$ - конечное множество (словарь) всех употребляемых в них термов, $T$ - конечное множество тем. Каждый документ $d \in D$ представляет собой последовательность $n_{d}$ термов $\left(w_{1}, \ldots, w_{n_{d}}\right)$ из словаря $W$. Примем гипотезу "мешка слов", согласно которой порядок термов в документе не важен. Обозначим через $n_{d w}$ число вхождений терма $w$ в документ $d$.

Коллекцию документов будем рассматривать как множество троек $(d, w, t) \in D \times W \times T$, выбранных случайно и независимо из дискретного распределения $p(d, w, t)$. При этом документы $d$ и термы $w$ являются наблюдаемыми переменными, темы $t$ являются латентными (скрытыми) переменными.

Примем гипотезу условной независимости $p(w \mid d, t)=p(w \mid t)$, согласно которой распределение термов в теме одинаково для всех документов. Тогда по формуле полной вероятности

$$
p(w \mid d)=\sum_{t \in T} p(w \mid d, t) p(t \mid d)=\sum_{t \in T} p(w \mid t) p(t \mid d)=\sum_{t \in T} \phi_{w t} \theta_{t d},
$$

где $\phi_{w t}=p(w \mid t)$ - неизвестное распределение термов в темах, $\theta_{t d}=p(t \mid d)$ - неизвестное распределение тем в документах. Условная вероятность $p(w \mid d)$ называется вероятностной тематической моделью документа, переменные $\phi_{w t}$ и $\theta_{t d}$ - параметры этой модели.

Задача вероятностного тематического моделирования заключается в том, чтобы найти параметры модели по эмпирическим данным $n_{d w}$. Для этого решается задача максимизации логарифма правдоподобия

$$
L(\Phi, \Theta)=\sum_{d \in D} \sum_{w \in d} n_{d w} \ln p(w \mid d)=\sum_{d \in D} \sum_{w \in d} n_{d w} \ln \sum_{t \in T} \phi_{w t} \theta_{t d} \rightarrow \max _{\Phi, \Theta}
$$

при ограничениях неотрицательности и нормировки:

$$
\phi_{w t} \geq 0, \quad \sum_{w \in W} \phi_{w t}=1, \quad \theta_{t d} \geq 0, \quad \sum_{t \in T} \theta_{t d}=1,
$$

где $\Phi$ и $\Theta$ - матрицы параметров $\phi_{w t}$ и $\theta_{t d}$ соответственно.

Задача (1.1) является некорректно поставленной задачей приближенного стохастического матричного разложения $\left(\frac{n_{d w}}{n_{d}}\right) \approx \Phi \Theta$, имеющей в общем случае бесконечное множество решений. Чтобы выбрать из него наиболее подходящее решение, вводятся дополнительные критерии - регуляризаторы $R_{i}(\Phi, \Theta) \rightarrow \max , i=1, \ldots, k$. В подходе ARTM (см. [11;13;14]) предлагается максимизировать взвешенную сумму всех регуляризаторов $R(\Phi, \Theta)=\sum_{i=1}^{k} \tau_{i} R_{i}(\Phi, \Theta)$ совместно с основным критерием правдоподобия:

$$
L(\Phi, \Theta)+R(\Phi, \Theta)=\sum_{d \in D} \sum_{w \in d} n_{d w} \log \sum_{t \in T} \phi_{w t} \theta_{t d}+\sum_{i=1}^{k} \tau_{i} R_{i}(\Phi, \Theta) \rightarrow \max _{\Phi, \Theta}
$$

- при тех же ограничениях неотрицательности и нормировки.

Наиболее известные тематические модели PLSA и LDA являются частными случаями регуляризации. В модели вероятностного латентного семантического анализа PLSA [5] регуляризация не используется, $R(\Phi, \Theta)=0$. В модели латентного размещения Дирихле LDA [2] регуляризатором является логарифм правдоподобия априорного распределения Дирихле

$$
R(\Phi, \Theta)=\sum_{t \in T} \sum_{w \in W}\left(\beta_{w}-1\right) \ln \phi_{w t}+\sum_{d \in D} \sum_{t \in T}\left(\alpha_{t}-1\right) \ln \theta_{t d}
$$


с гиперпараметрами $\beta_{w}, \alpha_{t}$, которые на практике обычно фиксируются, но могут и оптимизироваться с помощью специальных численных методов (см. [16]). В данной работе проблемы оптимизации гиперпараметров в критериях регуляризации, а также выбора коэффициентов регуляризации $\tau_{i}$, не рассматриваются.

Применение теоремы Каруша - Куна - Таккера позволяет выписать систему уравнений для стационарных точек оптимизационной задачи (1.2). Решение данной системы методом простых итераций приводит к ЕМ-подобному алгоритму, в котором на каждой итерации чередуются два шага: Е-шаг (expectation) и М-шаг (maximization).

На Е-шаге вычисляются значения условных вероятностей $p_{t d w}=p(t \mid d, w)$ по текущим значениям параметров $\phi_{w t}$ и $\theta_{t d}$ :

$$
p_{t d w}=\frac{\varphi_{w t} \theta_{t d}}{\sum_{s} \varphi_{w s} \theta_{s d}}
$$

Данное выражение совпадает с формулой Байеса, поскольку в силу гипотезы условной независимости $p(t \mid d, w)=\frac{p(w \mid t) p(t \mid d)}{p(w \mid d)}$.

На М-шаге по условным вероятностям тем $p_{t d w}$ для каждого терма в каждом документе вычисляются новые приближения параметров $\phi_{w t}$ и $\theta_{t d}$ и вспомогательные переменные $n_{d w t}$, $n_{w t}, n_{t d}, n_{t}, n_{d}, r_{w t}, r_{t d}$ :

$$
\begin{array}{rlrl}
n_{d w t} & =n_{d w} p_{t d w}, & & n_{t d} \\
n_{w t} & =\sum_{d \in D} n_{d w t}, & \sum_{w \in d} n_{d w t}, \\
n_{t} & =\sum_{w \in W} n_{w t}, & n_{d} & =\sum_{t \in T} n_{t d}, \\
r_{w t} & =\phi_{w t} \frac{\partial R}{\partial \phi_{w t}}, & r_{t d} & =\theta_{t d} \frac{\partial R}{\partial \theta_{t d}}, \\
\phi_{w t} & =\operatorname{norm}_{w \in W}\left(n_{w t}+r_{w t}\right), & \theta_{t d} & =\operatorname{norm}_{t \in T}\left(n_{t d}+r_{t d}\right),
\end{array}
$$

где $\underset{i \in I}{\operatorname{norm}}\left(x_{i}\right)=\frac{\left(x_{i}\right)_{+}}{\sum_{j \in I}\left(x_{j}\right)_{+}}-$операция нормировки, которая переводит произвольный числовой вектор $\left(x_{i}: i \in I\right)$ в дискретное вероятностное распределение, операция $\left(x_{i}\right)_{+}=\max \left(x_{i}, 0\right)$ называется положительной срезкой.

Вспомогательные переменные $n_{*}$ интерпретируются как оценки счетчиков: $n_{d w t}-$ число вхождений терма $w$ в документ $d$, связанных с темой $t ; n_{t d}$ - число всех термов в документе $d$, связанных с темой $t ; n_{w t}$ - число раз, когда терм $w$ был связан с темой $t$ во всей коллекции; $n_{t}$ - число термов, связанных с темой $t$ во всей коллекции; $n_{d}$ совпадает с длиной документа $d$.

Вспомогательные переменные $r_{w t}$ и $r_{t d}$ будем называть регуляризационными поправками. Заметим, что при $R=0$, т. е. в модели PLSA, $r_{w t}=0, r_{t d}=0, \phi_{w t}=\frac{n_{w t}}{n_{t}}, \theta_{t d}=\frac{n_{t d}}{n_{d}}$.

\section{2. Теорема о сходимости ЕM-алгоритма в ARTM}

Достаточные условия для сходимости обобщенного ЕМ-алгоритма как GEM алгоритма были сформулированы в [17]. Мы собираемся использовать те же методы доказательства, интерпретируя итерации ЕM-алгоритма ARTM как итерации GEM алгоритма.

Объединяя несколько теорем из [17] и адаптируя обозначения, нетрудно получить теорему, с помощью которой удобно доказывать сходимость ЕM-алгоритма в ARTM. 
Теорема 1. Пусть $\left\{\left(\Phi^{k}, \Theta^{k}\right)\right\}$ - траектория итерационного процесса, сгенерированная правилом $\left(\Phi^{k+1}, \Theta^{k+1}\right)=M\left(\Phi^{k}, \Theta^{k}\right)$, где $M$ - непрерывное преобразование пары стохастических матрии. Пусть функция $F(\Phi, \Theta)$ ограничена сверху и строго возрастает под действием $M$ на $(\Phi, \Theta)$. Тогда все предельные точки траектории $\left(\Phi^{k}, \Theta^{k}\right)$ являются стационарными точками F. Если также $\left\|\phi_{w t}^{k}-\phi_{w t}^{k+1}\right\| \rightarrow 0 u\left\|\theta_{t d}^{k}-\theta_{t d}^{k+1}\right\| \rightarrow 0$, а множество стационарных точек $F$ дискретно, то $\left(\Phi^{k}, \Theta^{k}\right)$ сходится $\kappa$ некоторой стационарной точке $F$.

О п р е д е л е н и е 1 . Регуляризатор $R$ является $\delta$-регулярным, если на итерациях ЕMалгоритма $\forall t \exists w: n_{w t}+r_{w t}>\delta$ и $\forall d \exists t: n_{t d}+r_{t d}>\delta$. Если регуляризатор обладает свойством $\delta$-регулярности при некотором $\delta>0$, то будем говорить, что регуляризатор сильно регулярен; при $\delta=0$ будем просто говорить, что он регулярен.

Регулярность гарантирует, что в операции norm не возникнет деления на нуль, т. е. итерации корректно определены. Сильная же регулярность позволяет утверждать, что преобразования, которые производятся на итерациях алгоритма, являются непрерывными по $(\Phi, \Theta)$. Это свойство легко выполняется на практике: если значение $n_{w t}+r_{w t}\left(\right.$ или $\left.n_{t d}+r_{t d}\right)$ становится меньше $\delta$, то вся тема (весь документ) исключается из модели и итерации продолжаются.

О п р е д е л е н и е 2. Регуляризатор $R$ сохраняет нуль, если на итерациях алгоритма из $n_{w t}=0$ следует $\phi_{w t}=0$ и из $n_{t d}=0$ следует $\theta_{t d}=0$.

Это определение формализует следующие свойство итерационного процесса: если на какойлибо итерации значение $\phi_{w t}$ стало равным нулю, то оно будет оставаться нулевым на последующих итерациях, и аналогично для $\theta_{t d}$. Для регуляризатора данное свойство легко проверяется аналитически. На практике многие регуляризаторы им обладают. Регуляризатор модели LDA, вообще говоря, не обладает данным свойством при $\beta_{w}>1$ или $\alpha_{t}>1$, так как при $n_{w t}=0$ вполне может оказаться, что $\phi_{w t}>0$. Однако при использовании ненулевой инициализации $\phi_{w t}$ значение $n_{w t}$ не может обратиться в нуль. Поэтому и для такого регуляризатора условие сохранения нуля выполняется.

О п р е д е л е н и е 3. Регуляризатор $R$ называется $\epsilon$-разреживающим, если на итерациях ЕМ-алгоритма $\phi_{w t}, \theta_{t d} \notin(0, \epsilon)$.

Некоторые регуляризаторы имеют неограниченную в окрестности нуля производную, в связи с чем при реализации ЕМ-алгоритма параметры, меньшие некоторого $\epsilon$, зануляются. Это приводит к тому, что значения в матрице параметров оказываются отделены от нуля. Именно эта особенность отражена в данном определении.

О п р е д е л е н и е 4. Регуляризатор $R$ корректный, если на итерациях ЕМ-алгоритма из $n_{d w}>0$ следует $p_{t d w}>0$ хотя бы для одной темы $t$.

Если модель дает нулевую оценку вероятности $p(w \mid d)=0$, при том что терм $w$ встречается в документе, $n_{d w}>0$, то логарифм правдоподобия становится неограниченным, $L \rightarrow-\infty$. На практике этого легко избежать, если использовать регуляризатор сглаживания фоновых тем (см. [13]). Он гарантирует, что для любого терма в любом документе найдется хотя бы одна тема с ненулевой вероятностью.

Введем вспомогательный функционал

$$
Q\left(\Phi, \Theta, \Phi^{\prime}, \Theta^{\prime}\right)=\sum_{d, w, t} n_{d w} p_{t d w}^{\prime} \ln \left(\phi_{w t} \theta_{t d}\right)+R(\Phi, \Theta), \quad p_{t d w}^{\prime}=\frac{\phi_{w t}^{\prime} \theta_{t d}^{\prime}}{\sum_{t} \phi_{w t}^{\prime} \theta_{t d}^{\prime}}
$$

Это стандартный прием при доказательстве сходимости GEM алгоритма. Изменения $Q$ на итерациях, как будет показано в дальнейшем, являются нижней оценкой для изменений $L+R$. Аналогичный функционал вводился в статьях [3;17].

Теорема 2. Пусть регуляризатор $R$ является дифберенцируемой функиией при $\phi_{w t}$, $\theta_{t d} \in(0,1]$, сохраняющей нуль, корректной, $\epsilon$-разрежсивающей $u \delta$-регулярной. Также допустим, что $Q\left(\Phi^{k+1}, \Theta^{k+1}, \Phi^{k}, \Theta^{k}\right) \geq Q\left(\Phi^{k}, \Theta^{k}, \Phi^{k}, \Theta^{k}\right)$, начиная с некоторой итерации $k$. 
Тогда последовательность $p_{t d w}^{k}$ сходится в смысле дивергениии Кульбака - Лейблера для любъх $d$ u w makux, чmo $n_{d w}>0$ :

$$
\mathrm{KL}\left(p_{t d w}^{k} \| p_{t d w}^{k+1}\right) \rightarrow 0 \quad \text { npu } \quad k \rightarrow \infty .
$$

Д о к а з а т е л ь с т в о. Поскольку регуляризатор сохраняет нуль, то, начиная с некоторой итерации, множество ячеек с нулевыми значениями в матрицах $\Phi$ и $\Theta$ стабилизируется и больше не будет изменяться. Это следует из того, что нулевое значение в ячейке не может стать на следующей итерации ненулевым, а множество всех ячеек конечно. Обозначим стабилизировавшееся множество ненулевых ячеек в матрицах $\Phi$ и $\Theta$ через $\Omega$. Поскольку регуляризатор $\epsilon$-разреживающий, значения $\Phi$ и $\Theta$ в позициях из $\Omega$ не могут быть менее $\epsilon$. Но $R-$ дифференцируемая функция при $\phi_{w t}, \theta_{t d} \in[\epsilon, 1]$, а значит, непрерывная и ограниченная.

Заметим, что $Q$ можно переписать следующим образом:

$$
Q\left(\Phi, \Theta, \Phi^{\prime}, \Theta^{\prime}\right)=L(\Phi, \Theta)+R(\Phi, \Theta)+\sum_{d, w, t} n_{d w} p_{t d w}^{\prime} \ln p_{t d w}
$$

На М-шаге $k$-й итерации были получены матрицы $\left(\Phi^{k+1}, \Theta^{k+1}\right)$.

По условию теоремы начиная с некоторой итерации, выполнено

$$
Q\left(\Phi^{k+1}, \Theta^{k+1}, \Phi^{k}, \Theta^{k}\right) \geq Q\left(\Phi^{k}, \Theta^{k}, \Phi^{k}, \Theta^{k}\right) .
$$

Подставим сюда вместо $Q$ его выражение по определению:

$$
\begin{gathered}
L\left(\Phi^{k+1}, \Theta^{k+1}\right)+R\left(\Phi^{k+1}, \Theta^{k+1}\right)+\sum_{d, w, t} n_{d w} p_{t d w}^{k} \ln p_{t d w}^{k+1} \\
\geq L\left(\Phi^{k}, \Theta^{k}\right)+R\left(\Phi^{k}, \Theta^{k}\right)+\sum_{d, w, t} n_{d w} p_{t d w}^{k} \ln p_{t d w}^{k},
\end{gathered}
$$

откуда имеем

$$
\Delta^{k}(L+R) \geq \sum_{d, w, t} n_{d w} p_{t d w}^{k} \ln \frac{p_{t d w}^{k}}{p_{t d w}^{k+1}}=\sum_{d, w} n_{d w} \operatorname{KL}\left(p_{d w}^{k} \| p_{d w}^{k+1}\right) \geq 0
$$

Равенство достигается, только если на итерации не произошло никаких изменений, что означает, что процесс сошелся в неподвижную точку. В обратном же случае $L+R$ строго увеличивается. Но это - ограниченная функция, значит, $L\left(\Phi^{k}, \Theta^{k}\right)+R\left(\Phi^{k}, \Theta^{k}\right)$ сходится при $k \rightarrow \infty$. Более того, $\mathrm{KL}\left(p_{t d w}^{k} \| p_{t d w}^{k+1}\right) \leq \Delta(L+R)^{k} \rightarrow 0$ при $n_{d w}>0$, что завершает доказательство.

Следствие 1. Если в дополнение к условиям теоремы 2 регуляризатор $R$ сильно регулярен, а $r_{w t} u r_{t d}$ непрерывны по параметрам модели, то

$$
\left|\phi_{w t}^{k}-\phi_{w t}^{k+1}\right| \rightarrow 0 \quad u \quad\left|\theta_{t d}^{k}-\theta_{t d}^{k+1}\right| \rightarrow 0 .
$$

Д о к а з а т е л ь с т в о. Согласно неравенству Пинскера (см. [10])

$$
\|A-B\|_{1} \leq 2 \sqrt{\operatorname{KL}(A \| B)} .
$$

Поэтому сходимость по KL-дивергенции влечет за собой сходимость по $l_{1}$ норме. Осталось заметить, что в условиях теоремы $2 \phi_{w t}$ и $\theta_{t d}$ являются непрерывными функциями от $p_{t d w}$. Следовательно, сходимость вторых влечет за собой сходимость первых.

Рассмотрим функцию $F(\Phi, \Theta)=L(\Phi, \Theta)+R(\Phi, \Theta)$, определенную для тех $\Phi$ и $\Theta$, у которых множество нулевых позиций матриц совпадает с множеством ненулевых позиций $\Omega$, стабилизировавшимся в ходе итераций. 
Следствие 2. В условиях следствия 1, если прочесс не сошелся в неподвиюную точку, все пределъные точки траектории $\left(\Phi^{k}, \Theta^{k}\right)$ являются стационарными точками $F$. Если же множество стационарных точек $F$ дискретно, то $\left(\Phi^{k}, \Theta^{k}\right)$ сходится $\kappa$ некоторой стационарной точке $F$.

Д о к а з а т е л ь с т в о. В условиях следствия 1 применение одной итерации ЕМ-алгоритма к матрицам $\Phi$ и $\Theta$ является непрерывным преобразованием. Также в ходе доказательства теоремы было установлено, что функция $F \equiv L+R$ строго возрастает на итерациях, если процесс не сошелся в неподвижную точку. Остается заметить, что остальные условия теоремы 1 тоже выполнены, если рассматривать все функции на области определения с ограничением на множество ненулевых позиций $\Omega$.

Таким образом, итерационный процесс EM-алгоритма в ARTM разбивается (в предположении увеличения $Q$ ) на два этапа: первый - это выбор множества позиций $\Omega$ ненулевых ячеек в матрицах $\Phi$ и $\Theta$, второй - окончательная оптимизация значений в этих ячейках. Первый этап можно рассматривать как дискретную оптимизацию структуры разреженности матриц $\Phi$ и $\Theta$ и подготовку их начальных приближений для второго этапа. Сходимость алгоритма происходит именно на втором этапе.

Следовательно, остается доказать монотонное увеличение функционала $Q$ на втором этапе ЕМ-алгоритма при фиксированном множестве $\Omega$.

\section{3. Изменение регуляризированного правдоподобия в ЕМ-алгоритме}

Важным условием сходимости алгоритма ARTM является неуменьшение значения $Q$ на М-шаге. Далее будут приведены оценки изменения функционалов $L, R$ и $Q$. Поскольку мы рассматриваем второй этап итерационного процесса, когда множество нулевых позиций в матрицах $\Phi$ и $\Theta$ не изменяется, положительную срезку в формулах можно опустить.

Введем функционал $\bar{Q}\left(\Phi, \Theta, \Phi^{\prime}, \Theta^{\prime}\right)=\sum_{d, w, t} n_{d w} p_{t d w}^{\prime} \ln \left(\phi_{w t} \theta_{t d}\right)$. Тогда $Q=\bar{Q}+R$.

Провести анализ суммарного изменения функционала $Q$ на М-шаге напрямую затруднительно. Поэтому предлагается разложить это преобразование на два этапа. Первый этап максимизация $\bar{Q}$ :

$$
\left\{\begin{aligned}
\phi_{w t} & =\underset{w \in W}{\operatorname{norm}}\left(n_{w t}\right), \\
\theta_{t d} & =\underset{t \in T}{\operatorname{norm}}\left(n_{t d}\right) .
\end{aligned}\right.
$$

Второй этап (назовем его регуляризационным преобразованием) - максимизация $R$ :

$$
\left\{\begin{aligned}
\phi_{w t} & =\operatorname{norm}_{w \in W}\left(n_{w t}+r_{w t}\right), \\
\theta_{t d} & =\operatorname{norm}_{t \in T}\left(n_{t d}+r_{t d}\right) .
\end{aligned}\right.
$$

Таким образом, изменения функционалов будут оцениваться отдельно на каждом этапе. На первом осуществляется переход в точку $\left(n_{w t} / n_{t}, n_{t d} / n_{d}\right)$, которая является точкой максимума функционала $\bar{Q}$, а на втором проводится максимизация $R$.

Введем еще один функционал и обозначения для его частных производных:

$$
\begin{gathered}
\bar{R}\left(\left(m_{w t}\right),\left(m_{t d}\right)\right)=R\left(\frac{m_{w t}}{\sum_{w} m_{w t}}, \frac{m_{t d}}{\sum_{t} m_{t d}}\right)=R\left(\frac{m_{w t}}{m_{t}}, \frac{m_{t d}}{m_{d}}\right) ; \\
g_{w t} \equiv \frac{\partial \bar{R}}{\partial m_{w t}}, \quad g_{t d} \equiv \frac{\partial \bar{R}}{\partial m_{t d}}, \quad \phi_{w t}=\frac{m_{w t}}{\sum_{w} m_{w t}}, \quad \theta_{t d}=\frac{m_{t d}}{\sum_{t} m_{t d}} .
\end{gathered}
$$


Как видим, функционал $\bar{R}$ определен на паре произвольных неотрицательных матриц размера $|W| \times|T|$ и $|T| \times|D|$. Он нормирует эти матрицы и применяет к ним регуляризатор $R$. Отметим, что при регуляризационном преобразовании $\bar{R}\left(n_{w t}, n_{t d}\right)=R\left(n_{w t} / n_{t}, n_{t d} / n_{d}\right)$.

Утверждение. Для $g_{w t} u g_{t d}$ выполнено

$$
\begin{aligned}
g_{w t} & =\frac{1}{m_{t}} \sum_{u \in W}\left(\frac{\partial R}{\partial \phi_{w t}}-\frac{\partial R}{\partial \phi_{u t}}\right) \phi_{u t}, \\
g_{t d} & =\frac{1}{m_{d}} \sum_{s \in T}\left(\frac{\partial R}{\partial \theta_{t d}}-\frac{\partial R}{\partial \theta_{s d}}\right) \theta_{s d} .
\end{aligned}
$$

Д о к а з а т е ль с т в о. В силу нормировки $\phi_{w t}=\frac{m_{w t}}{\sum_{w} m_{w t}}$

$$
\frac{\partial \phi_{u t}}{\partial m_{w t}}=\frac{\partial \frac{m_{u t}}{\sum_{v} m_{v t}}}{\partial m_{w t}}=\frac{\frac{\partial m_{u t}}{\partial m_{w t}}}{\sum_{v} m_{v t}}-\frac{m_{u t}}{\left(\sum_{v} m_{v t}\right)^{2}}=\frac{[u=w]}{m_{t}}-\frac{\phi_{u t}}{m_{t}}=\frac{1}{m_{t}}\left([u=w]-\phi_{u t}\right) .
$$

Следовательно,

$$
\frac{\partial \bar{R}}{\partial m_{w t}}=\sum_{u} \frac{\partial R}{\partial \phi_{u t}} \frac{\partial \phi_{u t}}{\partial m_{w t}}=\frac{1}{m_{t}}\left(\frac{\partial R}{\partial \phi_{w t}}-\sum_{u} \frac{\partial R}{\partial \phi_{u t}} \phi_{u t}\right)=\frac{1}{m_{t}} \sum_{u}\left(\frac{\partial R}{\partial \phi_{w t}}-\frac{\partial R}{\partial \phi_{u t}}\right) \phi_{u t} .
$$

Формула для $g_{d t}$ доказывается аналогично.

Теперь докажем основную теорему.

Теорема 3. Пусть величины $r_{w t} u r_{t d}$ на M-шаге рассчитываются в точках

$$
\frac{n_{w t}}{\sum_{w} n_{w t}} \quad u \frac{n_{t d}}{\sum_{t} n_{t d}}
$$

Тогда в ходе регуляризационного преобразования (3.1) без занулений элементов матрии угол между вектором изменений и градиентом $R$ острый, если градиент ненулевой.

Д о к аз а т е л ь с т о. Докажем утверждение для $\Delta n_{w t}$; для $\Delta n_{t d}$ доказательство будет аналогичным. При регуляризационном преобразовании без занулений $\Delta n_{w t}=\phi_{w t} \frac{\partial R}{\partial \phi_{w t}}$, поэтому с учетом утверждения получаем

$$
\left\langle\Delta n, \nabla \bar{R}\left(n_{w t}, n_{t d}\right)\right\rangle=\sum_{w, t, u} \frac{1}{n_{t}}\left(\frac{\partial R}{\partial \phi_{w t}}-\frac{\partial R}{\partial \phi_{u t}}\right) \frac{\partial R}{\partial \phi_{w t}} \phi_{w t} \phi_{u t} .
$$

В силу симметрии суммы выполнено

$$
\begin{gathered}
\sum_{w, t, u} \frac{1}{n_{t}}\left(\frac{\partial R}{\partial \phi_{w t}}-\frac{\partial R}{\partial \phi_{u t}}\right) \frac{\partial R}{\partial \phi_{w t}} \phi_{w t} \phi_{u t}=\sum_{w, t, u} \frac{1}{n_{t}}\left(\frac{\partial R}{\partial \phi_{u t}}-\frac{\partial R}{\partial \phi_{w t}}\right) \frac{\partial R}{\partial \phi_{u t}} \phi_{w t} \phi_{u t} \\
=\sum_{w, t, u} \frac{1}{n_{t}}\left(\frac{\partial R}{\partial \phi_{w t}}-\frac{\partial R}{\partial \phi_{u t}}\right)\left(-\frac{\partial R}{\partial \phi_{u t}}\right) \phi_{w t} \phi_{u t} \\
=\frac{1}{2}\left(\sum_{w, t, u} \frac{1}{n_{t}}\left(\frac{\partial R}{\partial \phi_{w t}}-\frac{\partial R}{\partial \phi_{u t}}\right) \frac{\partial R}{\partial \phi_{w t}} \phi_{w t} \phi_{u t}+\sum_{w, t, u} \frac{1}{n_{t}}\left(\frac{\partial R}{\partial \phi_{w t}}-\frac{\partial R}{\partial \phi_{u t}}\right)\left(-\frac{\partial R}{\partial \phi_{u t}}\right) \phi_{w t} \phi_{u t}\right) \\
=\frac{1}{2} \sum_{t, w, u} \frac{1}{n_{t}}\left(\frac{\partial R}{\partial \phi_{w t}}-\frac{\partial R}{\partial \phi_{u t}}\right)^{2} \phi_{w t} \phi_{u t}=\sum_{t, w<u} \frac{1}{n_{t}}\left(\frac{\partial R}{\partial \phi_{w t}}-\frac{\partial R}{\partial \phi_{u t}}\right)^{2} \phi_{w t} \phi_{u t} \geq 0 .
\end{gathered}
$$


Пусть здесь достигается равенство, тогда $\frac{\partial R}{\partial \phi_{w t}}=\frac{\partial R}{\partial \phi_{u t}}$ для всех $u$ и $w$. В данном случае

$$
\begin{aligned}
\frac{\partial \bar{R}}{\partial n_{w t}} & =\frac{1}{n_{t}}\left(\frac{\partial R}{\partial \phi_{w t}}-\sum_{u} \frac{\partial R}{\partial \phi_{u t}} \phi_{u t}\right)=\frac{1}{n_{t}}\left(\frac{\partial R}{\partial \phi_{w t}}-\sum_{u} \frac{\partial R}{\partial \phi_{w t}} \phi_{u t}\right) \\
& =\frac{1}{n_{t}}\left(\frac{\partial R}{\partial \phi_{w t}}-\frac{\partial R}{\partial \phi_{w t}} \sum_{u} \phi_{u t}\right)=\frac{1}{n_{t}}\left(\frac{\partial R}{\partial \phi_{w t}}-\frac{\partial R}{\partial \phi_{w t}}\right)=0 .
\end{aligned}
$$

Значит, градиент нулевой. Получили противоречие. Поэтому неравенство строгое и угол острый, что и требовалось доказать.

Ранее было показано (теорема 2), что при определенных ограничениях на регуляризатор занулений ячеек в матрицах $\Phi$ и $\Theta$ не будет, начиная с некоторой итерации. Таким образом, если коэффициенты регуляризации не слишком большие, то изменение $n_{w t}$ и $n_{t d}$ будет незначительным. Поэтому при регуляризационном преобразовании будет происходить увеличение $R$ в силу локального изменения вдоль градиента.

Теперь нужно объединить результаты двух этапов. В ходе первого этапа осуществляется переход в точку максимума $\bar{Q}$, поэтому, градиент $\bar{Q}$ в этой точке нулевой. Это означает, что в ней градиент $\bar{Q}+R$ сонаправлен с градиентом $R$, откуда следует, что на этапе регуляризационного преобразования происходит неуменьшение $\bar{Q}+R$.

Остается понять, как изменяется этот функционал на первом этапе. Есть риск, что при максимизации $\bar{Q}$ значение $Q$ может уменьшиться, поэтому при реализации алгоритма необходимо дополнительно проверять, что значение $Q$ увеличилось на итерации, и использовать новое значение $\Phi$ и $\Theta$ только, если увеличение произошло. Эта проверка строго гарантирует неуменьшение $Q$ на итерациях.

\section{4. Модификация М-шага}

Обычно в реализациях ЕM-алгоритма для ARTM (см. [1;4;12]) регуляризационные поправки $r_{w t}$ и $r_{t d}$ рассчитываются в точке $\left(\Phi^{k}, \Theta^{k}\right)$. В этом случае нет теоретических гарантий увеличения $Q$ на этапе регуляризационного преобразования. Поэтому алгоритм может сойтись в неподвижную точку отображения, а не в стационарную точку функционала $L+R$, из-за чего значение $L+R$ окажется субоптимальным.

Теорема 3 утверждает, что если рассчитывать $r_{w t}$ и $r_{t d}$ в точке $\left(\left(n_{w t}^{k} / n_{t}^{k}\right),\left(n_{t d}^{k} / n_{d}^{k}\right)\right)$, т. е. на основе величин, подсчитанных на М-шаге $k$-й итерации, то будут выполнены теоретические гарантии оптимальности.

Таким образом, обычные формулы М-шага для регуляризационных поправок

$$
r_{w t}^{k}=\phi_{w t}^{k-1} \frac{\partial R}{\partial \phi_{w t}}\left(\Phi_{w t}^{k-1}, \Theta_{t d}^{k-1}\right), \quad r_{t d}^{k}=\theta_{t d}^{k-1} \frac{\partial R}{\partial \theta_{t d}}\left(\Phi_{w t}^{k-1}, \Theta_{t d}^{k-1}\right)
$$

заменяются на модифицированные согласно теореме 3 :

$$
r_{w t}^{k}=\frac{n_{w t}^{k}}{n_{t}^{k}} \frac{\partial R}{\partial \phi_{w t}}\left(\frac{n_{w t}^{k}}{n_{t}^{k}}, \frac{n_{t d}^{k}}{n_{d}^{k}}\right), \quad r_{t d}^{k}=\frac{n_{t d}^{k}}{n_{d}^{k}} \frac{\partial R}{\partial \theta_{w t}}\left(\frac{n_{w t}^{k}}{n_{t}^{k}}, \frac{n_{t d}^{k}}{n_{d}^{k}}\right) .
$$

В следующем разделе будет проведено сравнение этих двух версий ЕМ-алгоритма на реальной текстовой коллекции.

\section{5. Эксперимент}

Согласно теореме 3 если рассчитывать регуляризационные поправки $r_{w t}$ и $r_{t d}$ не по матрицам $\Phi_{w t}$ и $\Theta_{t d}$ с предыдущей итерации, а по матрицам $\left(n_{w t}\right)$ и $\left(n_{t d}\right)$, то значение оптимизируемого функционала будет гарантированно увеличиваться на втором этапе итерационного 
Стандартная формула М-шага

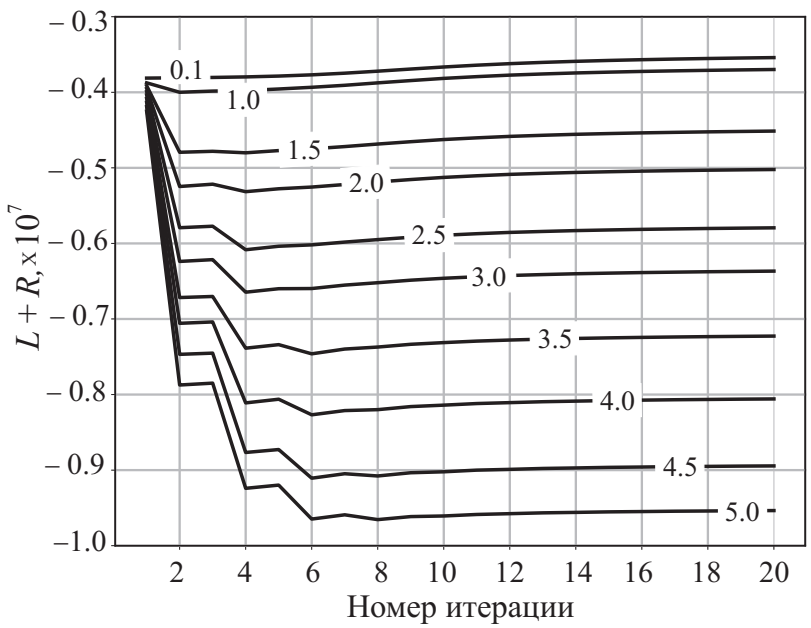

Модифицированная формула М-шага

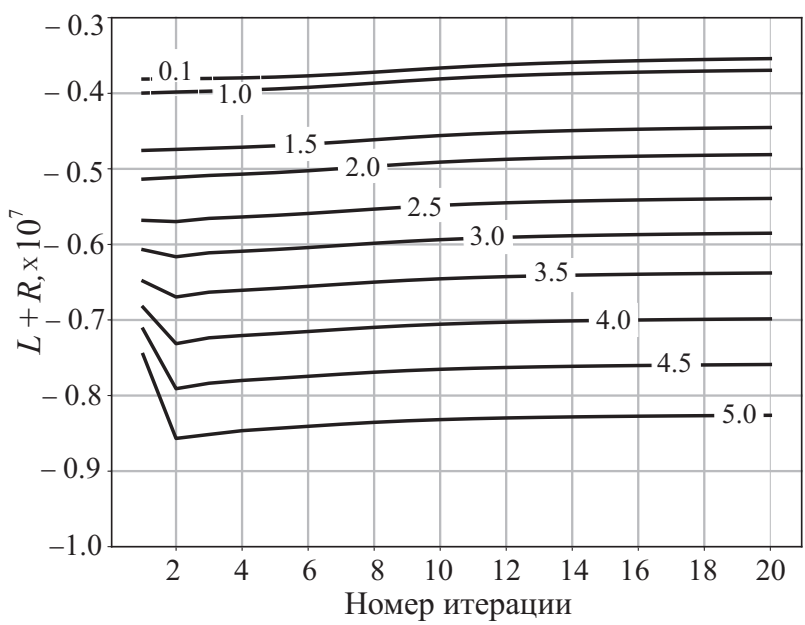

Изменение функционала $L+R$ на итерациях, $|T|=30$, при различных значениях коэффициента регуляризации $\tau$ (графики подписаны значениями $\tau$, уменьшенными в $10^{8}$ раз).

процесса. Ожидается, что это ускорит оптимизацию, позволяя за то же число итераций получать лучшие значения максимизируемого критерия.

Для экспериментальной проверки данного утверждения мы использовали лемматизированную коллекцию новостных сообщений на английском языке "20 NewsGroups" (см. [7]). Teматическая модель строилась ЕM-алгоритмом для ARTM, описанным в [13], с использованием регуляризатора декоррелирования [8]:

$$
R(\Phi)=-\frac{\tau}{|T|(|T|-1)} \sum_{t \neq s} \sum_{w \in W} \phi_{w t} \phi_{w s} .
$$

Указанный регуляризатор был выбран как один из наиболее часто используемых. Его максимизация позволяет увеличивать попарную различность тем как столбцов матрицы $\Phi$, улучшает интерпретируемость тем и способствует выделению фоновых тем с общей лексикой языка. При этом регуляризатор декоррелирования не имеет аналитического решения для задачи максимизации функционала $Q$ на М-шаге.

В эксперименте мы проверяли, как на итерациях алгоритма изменяется значение оптимизируемого функционала $L(\Phi, \Theta)+R(\Phi)$. Значения $\tau$ перебирались в таком интервале, чтобы абсолютная величина $R$ была соизмерима с абсолютным значением $L$ и регуляризатор оказывал заметное влияние на модель в процессе оптимизации. Сравнивались две версии М-шага: стандартная (4.1) и модифицированная (4.2).

\section{Итоговые значения функционала $L+R$ по окончании итераций}

\begin{tabular}{|c|c|c|c|}
\hline$\tau$ & $L+R$ стандарт & $L+R$ модификация & Увеличение $L+R, \%$ \\
\hline $10^{7}$ & -3536050 & -3536340 & -0.01 \\
\hline $10^{8}$ & -3693905 & -3691338 & 0.07 \\
\hline $1.5 \cdot 10^{8}$ & -4509247 & -4448501 & 1.35 \\
\hline $2.0 \cdot 10^{8}$ & -5018335 & -4808217 & 4.19 \\
\hline $2.5 \cdot 10^{8}$ & -5790283 & -5388187 & 6.94 \\
\hline $3.0 \cdot 10^{8}$ & -6363392 & -5848354 & 8.09 \\
\hline $3.5 \cdot 10^{8}$ & -7223361 & -6374974 & 11.75 \\
\hline $4.0 \cdot 10^{8}$ & -8055262 & -6982549 & 13.32 \\
\hline $4.5 \cdot 10^{8}$ & -8941616 & -7586618 & 15.15 \\
\hline $5.0 \cdot 10^{8}$ & -9532948 & -8259205 & 13.36 \\
\hline
\end{tabular}


На рисунке выше видно, что при стандартных формулах М-шага на первых итерациях происходит уменьшение функционала $L+R$, причем с ростом $\tau$ количество таких итераций растет. В то же время для модифицированного шага только одна итерация происходит с уменьшением $L+R$, далее наблюдается рост значений. Как и предполагалось, это позволяет получить заметно лучшие значения $L+R$ в точке, к которой сходится алгоритм. Их сравнение приводится в таблице. Также заметим, что чем больше $\tau$, т. е. чем сильнее воздействие регуляризатора на модель, тем существеннее предложенная модификация улучшает полученное решение.

\section{6. Заключение}

Данная работа закрывает проблему обоснования сходимости EM-алгоритма в ARTM при произвольном гладком критерии регуляризации. Полученные ограничения на регуляризатор не являются обременительными, легко проверяются и легко обеспечиваются программной реализацией. Весьма неожиданным оказался тот факт, что итерационный процесс, обычно используемый для построения регуляризованных моделей, в общем случае не гарантирует сходимости к стационарной точке. Модификация ЕМ-алгоритма, исправляющая этот недостаток, не требует дополнительных затрат времени или памяти. Она сводится к тому, что при вычислении регуляризационных поправок вместо текущих значений условных вероятностей $\phi_{w t}, \theta_{t d}$ следует подставлять их нерегуляризованные частотные оценки - ровно те, которые вычисляются в модели PLSA.

\section{СПИСОК ЛИТЕРАТУРЫ}

1. Apishev M.A., Vorontsov K.V. Learning topic models with arbitrary loss // Proc. of the 26th Conf. of FRUCT (Finnish-Russian University Cooperation in Telecommunications) Association. 2020. P. 30-37. doi: 10.23919/FRUCT48808.2020.9087559.

2. Blei D.M., Ng A.Y., Jordan M.I. Latent Dirichlet allocation // J. Mach. Learn. Res. 2003. Vol. 3. P. 993-1022.

3. Dempster A.P., Laird N.M., Rubin D.B. Maximum likelihood from incomplete data via the EM algorithm // J. Royal Statistical Society. Series B (methodological). 1977. Vol. 39, no. 1. P. 1-38.

4. Frei O.I., Apishev M.A. Parallel non-blocking deterministic algorithm for online topic modeling // Analysis of Images, Social networks and Texts (AIST'2016) - Communications in Computer and Information Science (CCIS). Cham: Springer International Publ., 2016. Vol. 661. P. 132-144. doi: 10.1007/978-3-319-52920-2_13.

5. Hofmann T. Probabilistic latent semantic indexing // Proc. of the 22nd Annual International ACM SIGIR Conf. on Research and Development in Information Retrieval. N Y: ACM, 1999. P. 50-57. doi: $10.1145 / 312624.312649$.

6. Kochedykov D.A., Apishev M.A., Golitsyn L.V., Vorontsov K.V. Fast and modular regularized topic modelling // Proc. of The 21st Conference of FRUCT (Finnish-Russian University Cooperation in Telecommunications) Association. The seminar on Intelligence, Social Media and Web (ISMW). (Helsinki, Finland, November 6-10, 2017). N Y: ACM, 2017. P. 182-193. doi: 10.23919/FRUCT.2017.8250181.

7. Lang K. 20 newsgroups [e-resource]. 2008. Data retrieved from the dataset's official website. URL: http://qwone.com/ jason/20Newsgroups/.

8. Tan Y., Ou Z. Topic-weak-correlated latent Dirichlet allocation // 7th International Symposium Chinese Spoken Language Processing (ISCSLP). 2010. P. 224-228. doi: 10.1109/ISCSLP.2010.5684906.

9. Tikhonov A.N., Arsenin V.Y. Solution of ill-posed problems. N Y etc.: John Wiley \& Sons, 1977. $258 \mathrm{p}$.

10. Topsøe F. Some inequalities for information divergence and related measures of discrimination // Information Theory, IEEE Transactions on. 2000. Vol. 46, no. 4. P. 1602-1609. doi: 10.1109/18.850703.

11. Vorontsov K.V. Additive regularization for topic models of text collections // Dokl. Math. 2014. Vol. 89, no. 3. P. 301-304. doi: 10.1134/S1064562414020185.

12. Vorontsov K.V., Frei O.I., Apishev M.A., Romov P.A., Suvorova M.A. BigARTM: Open source library for regularized multimodal topic modeling of large collections // Analysis of Images, Social 
Networks and Texts (AIST'2015) - Communications in Computer and Information Science (CCIS). Cham: Springer International Publ., 2015. Vol. 542. P. 370-381. doi: 10.1007/978-3-319-26123-2_36.

13. Vorontsov K.V., Potapenko A.A. Tutorial on probabilistic topic modeling: Additive regularization for stochastic matrix factorization // Analysis of Images, Social Networks and Texts (AIST'2014). Cham: Springer International Publ., 2014. Vol. 436. P. 29-46. doi: 10.1007/978-3-319-12580-0_3.

14. Vorontsov K.V., Potapenko A.A. Additive regularization of topic models // Machine Learning: Special Issue on Data Analysis and Intelligent Optimization with Applications. 2015. Vol. 101, no. 1. P. 303-323. doi: 10.1007/s10994-014-5476-6.

15. Vorontsov K.V., Potapenko A.A., Plavin A.V. Additive regularization of topic models for topic selection and sparse factorization // The Third International Symposium on Learning and Data Sciences (SLDS 2015). (April 20-22, 2015. Royal Holloway, University of London, UK.) Cham: Springer International Publ., 2015. P. 193-202. doi: 10.1007/978-3-319-17091-6_14.

16. Wallach H.M., Mimno D.M., McCallum A. Rethinking LDA: Why priors matter // Advances in Neural Information Processing Systems. Red Hook: Curran Associates, 2009. P. 1973-1981.

17. Wu C.J. On the convergence properties of the EM algorithm // The Annals of Statistics. 1983. Vol. 11, no. 1. P. 95-103. doi: 10.1214/aos/1176346060.

Ирхин Илья Александрович

Поступила 20.07.2020

После доработки 6.08.2020

Принята к публикации 17.08.2020

аспирант

Московский физико-технический институт

г. Москва

e-mail: ilirhin@gmail.com

Воронцов Константин Вячеславович

д.ф.-м.н., профессор РАН

зав. лаб. машинного интеллекта МФТИ

Московский физико-технический институт

г. Москва

e-mail: k.v.vorontsov@phystech.edu

\section{REFERENCES}

1. Apishev M.A., Vorontsov K.V. Learning topic models with arbitrary loss. In: Proceeding of the 26th Conf. of FRUCT (Finnish-Russian University Cooperation in Telecommunications) Association, 2020, pp. 30-37. doi: 10.23919/FRUCT48808.2020.9087559.

2. Blei D.M., Ng A.Y., Jordan M.I. Latent Dirichlet allocation. J. Mach. Learn. Res., 2003, vol. 3, pp. 993-1022.

3. Dempster A.P., Laird N.M., Rubin D.B. Maximum likelihood from incomplete data via the EM algorithm. J. Royal Statistical Society: Series B, 1977, vol. 39, no. 1, pp. 1-38.

4. Frei O. I., Apishev M. A. Parallel Non-blocking Deterministic Algorithm for Online Topic Modeling. In: Ignatov D. et al. (eds), Analysis of Images, Social Networks and Texts (AIST'2016), 2017, Communications in Computer and Information Science, vol. 661, Cham: Springer, pp. 132-144. doi: 10.1007/978-3-319-52920-2_13.

5. Hofmann T. Probabilistic latent semantic indexing. In: Proc. of the 22nd Annual International ACM SIGIR Conf. on Research and Development in Information Retrieval. N Y: ACM, 1999, pp. 50-57. doi: $10.1145 / 312624.312649$.

6. Kochedykov D.A., Apishev M.A., Golitsyn L.V., Vorontsov K.V. Fast and modular regularized topic modelling. In: Proc. of the 21st Conf. of FRUCT (Finnish-Russian University Cooperation in Telecommunications) Association. The seminar on Intelligence, Social Media and Web (ISMW), Helsinki, Finland, November 6-10, 2017, 2017, pp. 182-193. doi: 10.23919/FRUCT.2017.8250181.

7. Lang K. 20 newsgroups. 2008. Data retrieved from the dataset's official website. Available at http://qwone.com/ jason/20Newsgroups/. 
8. Tan Y., Ou Z. Topic-weak-correlated latent Dirichlet allocation. In: 7th International Symposium Chinese Spoken Language Processing (ISCSLP). 2010, pp. 224-228. doi: 10.1109/ISCSLP.2010.5684906 .

9. Tikhonov A.N., Arsenin V.Ya. Solutions of ill-posed problems. N Y etc.: John Wiley \& Sons, 1977, 258 p. ISBN: 0-470-99124-0.

10. Topsøe F. Some inequalities for information divergence and related measures of discrimination. IEEE Transactions on Information Theory, 2000, vol. 46, no. 4, pp. 1602-1609. doi: 10.1109/18.850703.

11. Vorontsov K.V. Additive regularization for topic models of text collections. Dokl. Math., 2014, vol. 89, no. 3, pp. 301-304. doi: 10.1134/S1064562414020185.

12. Vorontsov K.V., Frei O.I., Apishev M.A., Romov P.A., Suvorova M.A. BigARTM: Open Source Library for Regularized Multimodal Topic Modeling of Large Collections. In: Khachay M., Konstantinova N., Panchenko A., Ignatov D., Labunets V. (eds), Analysis of Images, Social Networks and Texts, 2015, Communications in Computer and Information Science, vol. 542, Cham: Springer, pp. 370-381. doi: 10.1007/978-3-319-26123-2_36.

13. Vorontsov K.V., Potapenko A.A. Tutorial on Probabilistic Topic Modeling: Additive Regularization for Stochastic Matrix Factorization. In: Ignatov D., Khachay M., Panchenko A., Konstantinova N., Yavorsky R. (eds), Analysis of Images, Social Networks and Texts, 2014, Communications in Computer and Information Science, vol. 436, Cham: Springer, pp. 29-46. doi: 10.1007/978-3-319-12580-0_3.

14. Vorontsov K.V., Potapenko A.A. Additive regularization of topic models. Machine Learning, 2015, vol. 101, no. 1, pp. 303-323. doi: 10.1007/s10994-014-5476-6 .

15. Vorontsov K.V., Potapenko A.A., Plavin A.V. Additive Regularization of Topic Models for Topic Selection and Sparse Factorization. In: Gammerman A., Vovk V., Papadopoulos H. (eds), Statistical Learning and Data Sciences, 2015, Lecture Notes in Computer Science, vol. 9047, Cham: Springer, pp. 193-202. doi: 10.1007/978-3-319-17091-6_14.

16. Wallach H.M., Mimno D.M., McCallum A. Rethinking LDA: why priors matter. In: Proceedings of the 22nd International Conference on Neural Information Processing Systems (NIPS'09). Red Hook: Curran Associates, pp. 1973-1981.

17. Wu C.J. On the Convergence Properties of the EM Algorithm. The Annals of Statistics, 1983, vol. 11, no. 1, pp. 95-103. doi: 10.1214/aos/1176346060.

Received July 20, 2020

Revised August 6, 2020

Accepted August 17, 2020

Funding Agency: The work was performed within the project "Text mining tools for big data" according to the program of the Competence Center of the National Technological Initiative "Center for Big Data Storage and Processing" supported by the Ministry of Science and Higher Education of the Russian Federation under the agreement between Moscow State University and the NTI Fund of August 15, 2019, no. 7/1251/2019. This work was also partially supported by the Russian Foundation for Basic Research (project no. 20-07-00936).

Konstantin Vyacheslavovich Vorontsov, Dr. Phys.-Math. Sci, Prof., Moscow Institute of Physics and Technology (State University), Dolgoprudny, 141701 Russia, e-mail: k.v.vorontsov@phystech.edu .

Il'ya Aleksandrovich Irkhin, doctoral student, Moscow Institute of Physics and Technology (State University), Dolgoprudny, 141701 Russia, e-mail: ilirhin@gmail.com.

I. A. Irkhin, K. V. Vorontsov. Convergence of the algorithm of additive regularization of topic models, Trudy Instituta Matematiki i Mekhaniki URO RAN, 2020, vol. 26, no. 3, pp. 56-68. 